\title{
Itinerarios de las políticas culturales públicas en Rosario, Argentina
}

\author{
LAURA ANA CARDINI
}

El propósito de este artículo es exponer aspectos centrales de una investigación socioantropológica sobre las políticas culturales en la ciudad de Rosario, Argentina. La creación de la primera área cultural estatal en dicha ciudad se remonta a finales de la década de 1930, y principalmente a mediados de la década de 1980 se propagan las dependencias en esta materia. La década de 1990 es un momento de ampliación de las nociones de cultura y los territorios de la acción cultural, con la incorporación de nuevos actores y modificaciones en la institucionalidad del espacio estatal. Este escrito se sustenta en una perspectiva teórico-metodológica para el análisis de las normativas municipales y en entrevistas a los trabajadores de las áreas involucradas.

PALABRAS CLAVE: cultura, institucionalización, ciudad, Rosario, conocimiento socioantropológico

\section{Itineraries of Public Cultural Policies in Rosario City, Argentina}

The purpose of this article is to expose central aspects of socioanthropological research on cultural policies in Rosario city, Argentina. The creation of the first state cultural area in this city dates back to the late 1930s, mainly in the mid1980s, dependencies were propagated in this area. The decade of 1990 is a moment of extension of the notions of culture and territories of cultural action, with the incorporation of new actors and modifications in the institutionality of the state space. This paper is based on a theoretical-methodological perspective in the analysis of municipal regulations and interviews to workers in the areas involved.

KEYWORDS: culture, institutionalization, city, Rosario, socioanthropological knowledge 


\section{Introducción}

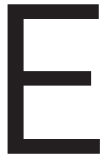

n la década de 1960 se registra una construcción institucional del área cultural (Miceli, 1987) y en la siguiente puede hablarse de un ámbito con especificidad propia dentro de los Estados que tienen a la cultura como espacio de intervención. Desde la década de 1980, diversos autores debaten en torno al lugar de la cultura y la política en Latinoamérica y las posibilidades de reflexionar de modo crítico sobre las acciones en esta materia en distintos contextos nacionales (García, 1987; Bonfil, 1987; Landi, 1987; Brunner, 1987). ${ }^{1}$ Se reconoce la importancia de un abordaje interdisciplinar para reflexionar sobre las políticas culturales y el patrimonio, la producción cultural, la identidad y la diversidad (Arantes, 1984; García, 1987; Ceballos, 1989; Rotman, 1996; Bayardo, 2000; 2010; Yúdice y Miller, 2004). Los autores alertan acerca de la multiplicidad de aproximaciones a la noción de políticas culturales, que se corresponden con las relaciones de trabajo de los propios autores con esta área específica y las tradiciones disciplinares de cada contexto nacional (Ochoa, 2002). Los contenidos teóricos, metodológicos y políticos atribuidos a sus términos responden a diferentes maneras mediante las cuales instituciones, intelectuales y organizaciones se han apropiado de la cultura como campo organizativo para articular transformaciones simbólicas, sociales y políticas (Ochoa, 2002). Entre los desafíos de este campo está la pregunta acerca de las relaciones y articulaciones entre cultura y Estado, cultura y mercado, cultura y creadores

1 Mencionamos algunos de los debates más significativos respecto de las políticas culturales en el ámbito latinoamericano para entender la experiencia en la que se enfoca el presente trabajo: las políticas culturales municipales de Rosario. En otros escritos (Cardini, 2013) hemos profundizado en los núcleos teórico-metodológicos que permiten ubicar la discusión acerca de las políticas culturales y sus articulaciones contemporáneas: el viraje de la cultura hacia la mercantilización (Aguilar, 2005); la cultura como recurso (Yúdice, 2002); el papel de la cultura en la promoción de las ciudades (Zukin, 1995); las políticas culturales y las producciones culturales urbanas (Rotman, 1996), por nombrar algunas. 
(Chaui, 2008), o también, en términos de la sociedad civil (Vich, 2014), entendida como un agente fundamental de las políticas culturales en la conquista de derechos ciudadanos.

En Argentina son variados los desarrollos teórico-metodológicos en el campo de la antropología y disciplinas afines, tales como la historia, la comunicación, la filosofía, entre otras, con reflexiones surgidas de encuentros entre investigadores y en los propios ámbitos de gestión de las políticas culturales. ${ }^{2}$ Asimismo, nuevos organigramas institucionales en diversos niveles del Estado -municipal, provincial y nacional - plantean un movimiento significativo para este campo.

En nuestro trabajo consideramos las políticas culturales como el conjunto de intervenciones y actuaciones que se generan tanto desde los ámbitos estatales como entre los distintos actores implicados, los cuales, en una dialéctica con las primeras, pueden reforzarlas, rechazarlas o confrontarlas (García, 1987; Achilli, 1998). Tenemos en cuenta un sentido estructural institucional respecto de las tendencias y formulaciones de las políticas públicas locales, así como de las trayectorias de los sujetos involucrados en las gestiones culturales, en el que los límites entre el Estado y sus entrelazamientos con los movimientos culturales no son absolutos (Landi, 1987). Esta perspectiva de las políticas culturales destaca la agencia, los intereses contradictorios, los posicionamientos desiguales y las disputas de poder de los actores, ya sea como pertenecientes a lo estatal, lo comunitario y lo privado, o como integrantes de colectivos artísticos y movimientos sociales, entre otros (Crespo, Morel y Ondelj, 2015).

En este artículo se parte de una perspectiva socioantropológica para el abordaje de las políticas públicas culturales en la ciudad de Rosario (Cardini, 2011), que se asienta en los desarrollos teóricometodológicos en el campo de las políticas culturales en el contexto latinoamericano y argentino, sistematizaciones de normativas locales y entrevistas a funcionarios y trabajadores de las áreas involucradas. ${ }^{3}$ Si bien la creación de la primera área cultural específica se remonta a 1937, recién a partir de 1983 - con la apertura democrática — se propagaron numerosas dependencias en esta materia. Arribamos a una caracterización de las políticas culturales locales en el contexto de la apertura democrática, para comprender los procesos de institucionalización de las áreas culturales de la ciudad y de los que vendrán después. En ese sentido, a partir de la década de 1980 identificamos tres momentos clave de las políticas culturales en Rosario, que nos orientan para reconstruir el devenir de la articulación entre cultura y política corporizada en las transformaciones del organigrama institucional y normativo: la década de 1980 y la apertura democrática; la década de 1990 y la nueva jerarquía del área; y las primeras décadas del siglo XXI, con la ampliación de las dependencias culturales. Ese derrotero de la cultura y la política estatal permite indagar en las continuidades y rupturas de las concepciones y prácticas de la cultura, lo público y la ciudad, y a la vez arribar a las razones del crecimiento exponencial de las áreas estatales de Rosario. Esta periodización constituye una herramienta para comprender las políticas culturales en esa ciudad y vislumbrar la década de 1990 como el momento de mayor ampliación de las nociones de cultura y los territorios de la acción cultural, con la incorporación de nuevos actores en el espacio estatal y modificaciones sustanciales en su institucionalidad. Se trata de un proceso paradójico, que deja al descubierto tensiones y modos específicos en los que se

Entre 2006 y 2013 se realizaron cuatro congresos nacionales de cultura, organizados por la Secretaría de Cultura de la Nación -actual Ministerio de Cultura de la Nación-.

3 Para este artículo retomamos algunas entrevistas en profundidad con funcionarios y trabajadores de áreas culturales locales de 1984 a 2004. A todos ellos extendemos un gran agradecimiento por compartir sus experiencias de vida y brindar información inestimable para el desarrollo de este estudio. 
expresaron las tendencias hegemónicas de la década neoliberal en Argentina, en el contexto particular de la ciudad de Rosario.

Por lo expuesto, destinamos un apartado a introducir elementos de la institucionalización de las áreas culturales en Argentina, en el que se recuperan las sistematizaciones de diversos autores argentinos y latinoamericanos. Luego, presentamos las transformaciones ocurridas en las dependencias estatales culturales de Rosario y señalamos momentos clave por medio del análisis de normativas y entrevistas. Más adelante, arribamos a una lectura de las concepciones de lo cultural y el Estado desde la perspectiva de sus funcionarios y trabajadores, que permite comprender las dinámicas de expansión del horizonte de lo cultural. Al final del artículo, retomamos algunos de los principales tópicos del itinerario de las políticas culturales públicas en Rosario y dejamos abierta la reflexión sobre el porvenir de dichas políticas en Argentina.

\section{Lo cultural como esfera de las políticas públicas en Argentina ${ }^{4}$}

Los antecedentes de institucionalización de las políticas públicas en materia cultural en Argentina se remontan a la década de 1930, con la creación de una Comisión Nacional de Cultura en 1933, conformada por representantes de diversos organismos públicos relacionados con asuntos culturales y educativos del país, bajo el gobierno de Agustín Justo (19321938), quien llegó al poder por medio de elecciones fraudulentas. Este organismo oficial comenzó a funcionar en 1935 y estuvo vigente hasta mediados de la década de 1950 (Niño, 2007). En esos años primaba una visión tradicionalista que asociaba la esencia de la identidad nacional con la tierra y la sangre, enlazada a las grandes instituciones, como el Ejército, la Iglesia, la familia y la propiedad (García, 1987). Desde la órbita del Ministerio de Justicia e
Instrucción Pública, en 1948, esa misma cartera se disgregó en dos ministerios, de Justicia y de Educación, y derivó a su vez en la creación de una Subsecretaría de Cultura (Lifschitz, 2008).

Con el gobierno de Juan Domingo Perón (1946-1955), en 1946 se creó la Secretaría de Cultura de la Nación. Las orientaciones de las políticas culturales durante los años del peronismo se caracterizaron por una visión sustancialista de la cultura alojada en el Estado; en él se condensaban los valores nacionales y se ordenaba, reunía y regulaba la sociedad. Aquí, el Estado asumía el control y la cohesión de la identidad nacional, que tenía al pueblo como el destinatario de la acción del gobierno, subordinado a los intereses de la nación. Las actividades dirigidas a cohesionar al pueblo y a ciertos sectores de la burguesía nacional, en oposición a la oligarquía, favorecieron el desarrollo de producciones culturales subalternas, como el tango y la poesía popular, y la creación de industrias culturales bajo la protección del Estado, en radiodifusión y cine (García, 1987).

El golpe de Estado en 1955 puso fin, entre otras cosas, al cuestionamiento de las estructuras de dominación y las medidas orientadas a reivindicar a las culturas populares. La Revolución Libertadora, nombre que recibió la dictadura cívico-militar que derrocó al presidente Perón, comenzó un proceso de repliegue de las expresiones artísticas y culturales vinculadas al periodo anterior. De la alternancia entre gobiernos desarrollistas - Arturo Frondizi (1958-1962) - y dictaduras militares - Pedro Aramburu (1966-1970)—, a finales de la década de

4

Para la reconstrucción del contexto argentino hemos seguido principalmente los planteamientos de Néstor García Canclini (1987) en relación con la legitimación política y la edificación del Estado-nación. Asimismo, en este apartado se retoma parte de nuestras reflexiones en el marco de un contrapunto con experiencias en el ámbito latinoamericano, que desarrollamos en otros trabajos (Cardini y Madrigal, 2018). 
1950 surgieron nuevos organismos abocados a las artes y demás expresiones culturales, como el Fondo Nacional de las Artes, que marcaron líneas de trabajo con orientación folclórica.

Entre 1976 y 1983 se sucedió la última dictadura militar, denominada Proceso de Reorganización Nacional, que se caracterizó por la reducción del gasto público en educación y cultura, y el avance de la transnacionalización económica y cultural, en un contexto de desocupación y empobrecimiento de salarios. En el campo cultural, la censura se expresó en los ámbitos artísticos, culturales e intelectuales por medio de diversos mecanismos: listas negras, persecuciones, desapariciones y cercenamiento de las producciones artísticas, educativas y de investigación científica.

El contexto posterior a los golpes de Estado atravesados por los países latinoamericanos constituyó un momento clave de las políticas culturales, con el retiro de la intervención estatal y las censuras sobre ámbitos de la creación artística y cultural. En la transición y apertura de la década de 1980, en Argentina, se sucedieron cambios en el campo cultural, con fuerte orientación hacia la "deselitización de la cultura” (Durham, 1984). Mientras tanto, los movimientos de la sociedad civil y los grupos de artistas trabajaban en los intersticios de la dictadura para ampliar las creaciones artísticas hacia diversos sectores. Connotaciones asociadas a las ideas de participación popular y pluralismo se trasladaron a las producciones culturales. La década de 1980 fue una bisagra en las políticas culturales de la democracia.

Las políticas culturales de este periodo estuvieron asociadas a transformaciones globales producto del cambio de régimen político. Luego del retiro de la censura — proceso lento y complejo-, se plantearon otras interrogantes vinculadas a cómo debía intervenir el Estado en el campo cultural (Landi, 1987).

Durante los años de redemocratización política del país, se generó un conjunto de planes culturales municipales, provinciales y nacionales, que sentó "las bases de discusión y confrontación de un país que se fue transformando subterráneamente en el silencio del autoritarismo" (Landi, 1987: 156). En esta dirección, el gobierno de Raúl Alfonsín (19841989) propuso un Plan Nacional de Cultura y su Programa de Democratización de la Cultura, el cual apelaba al descubrimiento y la defensa de la pluralidad de identidades, la descentralización del poder, la mejor distribución de los bienes culturales y el protagonismo de todos los habitantes de la nación; su retórica introdujo el derecho a la cultura (Landi, 1987). Este periodo involucró todo un conjunto de leyes de protección y fomento de la actividad cultural y las diversas actividades comerciales e industriales comprometidas en ellas. No obstante, el plan se asentó en las estructuras burocráticas heredadas del régimen anterior, cuyas atribuciones y funciones no permitieron la adecuación de esos enunciados en todas las dimensiones de su implementación. Ese proceso supuso una enunciación de principios, más que una política pública que alcanzara los distintos contextos provinciales y municipales.

La década de 1990 estuvo marcada por medidas de corte neoliberal, que introdujeron ajustes macroeconómicos y reformas institucionales, tales como la descentralización de responsabilidades y funciones del gobierno nacional hacia las provincias, la privatización de empresas públicas y las reducciones en los gastos referidos a programas sociales, culturales, educativos y científicos. Si bien en 1993 se había arribado al Plan Federal de Cultura, éste se aproximaba más a la compilación de las conclusiones de encuentros de cultura realizados por el organismo que a la formulación de un verdadero plan de acción nacional (Getino, 1995). Durante los dos periodos presidenciales de Carlos Saúl Menem (1989-1993; 1994-1999), las políticas neoliberales impactaron de lleno en el país y en 2001 se produjo el estallido de la crisis económica, política e institucional que caracterizó el comienzo del siglo XXI, bajo el gobierno radical de Fernando de la Rúa (1999-2001). 
A la crisis institucional de 2001, en la que en un breve lapso se sucedieron varios presidentes provisionales, le siguió el proceso electoral que llevó al poder a los gobiernos peronistas de Néstor Kirchner (2003-2007) y Cristina Fernández de Kirchner (2007-2015), en los cuales se impulsaron políticas con tendencia al rechazo al neoliberalismo y los tratados de libre comercio en la región, la defensa de procesos de integración latinoamericana, como el Mercado Comercial del Sur, la reivindicación de los derechos humanos, entre otras. Lo cultural cobró relevancia en términos de derechos culturales, de la mano de acciones, programas y nuevos organigramas institucionales. Fue muy importante la aprobación de la Ley de Servicios de Comunicación Audiovisual, que regularía esos servicios en todo el territorio nacional.

Si en la década de 1980 las cuestiones centrales pasaban por las libertades, en las primeras décadas del siglo XXI el énfasis se volcó a la construcción de igualdades, marcadas por el acento en la perspectiva de los derechos, con medidas tendentes a la ampliación de las ciudadanías (Raggio, 2015).

Asimismo, se desarrollaron procesos de institucionalización del sector cultural mediante la creación de áreas y adecuación de sus funciones. En 2010 se conformó y reglamentó el Consejo Federal de Cultura y en 2014 se creó el Ministerio de Cultura de la Nación, con la consigna de una cultura pública.

En 2015, las elecciones democráticas llevaron al gobierno al empresario Mauricio Macri (2015-2019), al frente de una coalición política que congregaba a sectores de la derecha política argentina. Entre las primeras medidas tomadas por esa gestión, se modificaron — por un decreto de necesidad y urgencialos componentes fundamentales de la Ley de Medios aprobada por el gobierno anterior, además de disolver su autoridad federal de aplicación. En el ámbito del Ministerio de Cultura de la Nación se produjeron modificaciones en la estructura del organismo y las directivas apuntaron a la vinculación privada y empresarial.

\section{Lo cultural en Rosario}

La ciudad de Rosario está ubicada en la zona sur de la provincia de Santa Fe, en el centro-este del país, e históricamente ha ocupado un lugar destacado en términos económicos y productivos. En la actualidad, cuenta con alrededor de un millón de habitantes y por la densidad de su población representa la tercera parte de la población de la provincia. ${ }^{5}$

Sin una fecha precisa, los comienzos de Rosario se remontan a un primer núcleo de población sobre la costa oeste del río Paraná, alrededor de una capilla que se instaló en 1731, en lo que se llamó Pago de los Arroyos. La fecha más antigua de los asentamientos en la zona se remonta a la presencia de una estancia llamada Concepción de los Arroyos en 1689 (Álvarez, 1998). Durante la segunda mitad del siglo XIX, la Villa del Rosario creció en forma abrumadora, producto del aporte inmigratorio masivo que transformó su estructura socioeconómica (Naranjo, 1973). En ese periodo, además, se convirtió en una ciudad portuaria, intermediaria entre un interior que comenzaría a desarrollar las actividades agropecuarias y un exterior que demandaba alimentos y productos de origen primario para sus industrias (Hardoy, 1985).

En 1852 se consolidaron política y económicamente las instituciones y Rosario fue declarada ciudad de manera oficial. A principios y mediados del siglo XIX, las asociaciones con fines culturales fueron significativas; las lideraban miembros de familias burguesas de la ciudad, con cierto perfil de mecenazgo, que trabajaban en relación con el poder

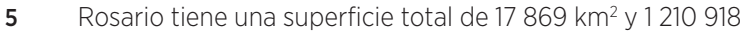
habitantes, según las cifras del último censo (INDEC, 2011). 
político e instalaron la expresión de lo público en la dinámica de las artes y la historia (Fernández y Armida, 2000). Esta articulación entre las esferas privadas y públicas acompañó el desenvolvimiento del campo cultural de la ciudad en relación con la formación de distintas instituciones, como teatros, museos, bibliotecas, salones de arte y cines, principalmente en las décadas de 1920, 1930 y 1940. De ahí que los museos que se crearon a comienzos del siglo XX, como el de Bellas Artes (1937) y el Histórico Provincial (1939), configuraran sus patrimonios iniciales por medio de la donación de colecciones familiares de la burguesía rosarina.

La primera dependencia estatal abocada a lo cultural se registró en 1937, con la Dirección de Cultura, dependiente de la Secretaría de Gobierno Municipal, que funcionó en el edificio del Museo Municipal de Bellas Artes Juan B. Castagnino.

Asimismo, más allá del radio de las instituciones, la producción cultural impulsada por movimientos y grupos de artistas plásticos, escritores, músicos y dramaturgos fue prolífica (Cardini, 2013). Salvo la mencionada Dirección de Cultura creada a finales de la década de 1930, no será sino hasta los años ochenta cuando se instituyan áreas específicas de lo cultural como una esfera de las políticas del Estado municipal.

\section{La década de 1980 y la reapertura democrática}

Durante la última dictadura militar en Argentina (1976-1983), el campo cultural suponía una arena de disputa ideológica "ya que a juicio de los militares ése era el espacio fundamental en el que se generaba la "subversión"” (Guevara, 2000: 246). El foco estaba en el control de todas las expresiones por medio de medidas como la programación de las radios y emisoras televisivas por parte del Estado, mecanismos de censura en la publicación de libros, diarios y revistas, la confección de listas negras, el exilio de grupos importantes de artistas e intelectuales, hasta la desaparición de algunos de ellos. La supervisión de cada una de las manifestaciones artísticas y culturales era efectuada por una Comisión Calificadora de Espectáculos Públicos e Impresos Literarios, integrada por miembros de la Liga de la Decencia, la Liga de Madres de Familia, la Municipalidad de Rosario y el Juzgado de Menores (Guevara, 2000; Logiódice, 2012).

Respecto del impacto de la dictadura militar en la ciudad, si bien la censura y las restricciones sobre las manifestaciones culturales y los medios de comunicación atravesaron todo ese periodo, se puede hablar de cierta distensión en los controles a los medios de comunicación hacia 1982, lo que condujo a una serie de cambios (Águila, 2000); entre ellos, la edición de un nuevo Diario Rosario y la incorporación de diferentes ciclos en la programación de los canales locales.

Asimismo, experiencias en el campo de la música, en el ámbito del teatro o en prácticas artísticas experimentales más radicales dieron muestras de un importante movimiento que empujó — aunque no de manera orgánica - para que las producciones culturales se propagaran en distintos ámbitos de la ciudad. De hecho, la participación de los grupos tuvo incidencia en lo que más tarde fueron las acciones culturales de la democracia, al trabajar, en ocasiones de modo conjunto, en el diseño y programación de actividades novedosas en zonas antes relegadas del horizonte de actividades culturales estatales.

En 1983, con Horacio Usandizaga (19831989) al frente del gobierno de la ciudad, perteneciente a la Unión Cívica Radical, la antigua Dirección de Cultura municipal pasó a ser una subsecretaría dependiente de la Secretaría de Gobierno y Cultura, a cargo de Rafael Ielpi (1984-1989), un reconocido escritor de la ciudad. ${ }^{6}$ Tal como expresa Gustavo Guevara: “[con la] democracia podría

6

De diciembre de 1983 a febrero de 1984, el área cultural estuvo a cargo del contador Martín Navarro. 


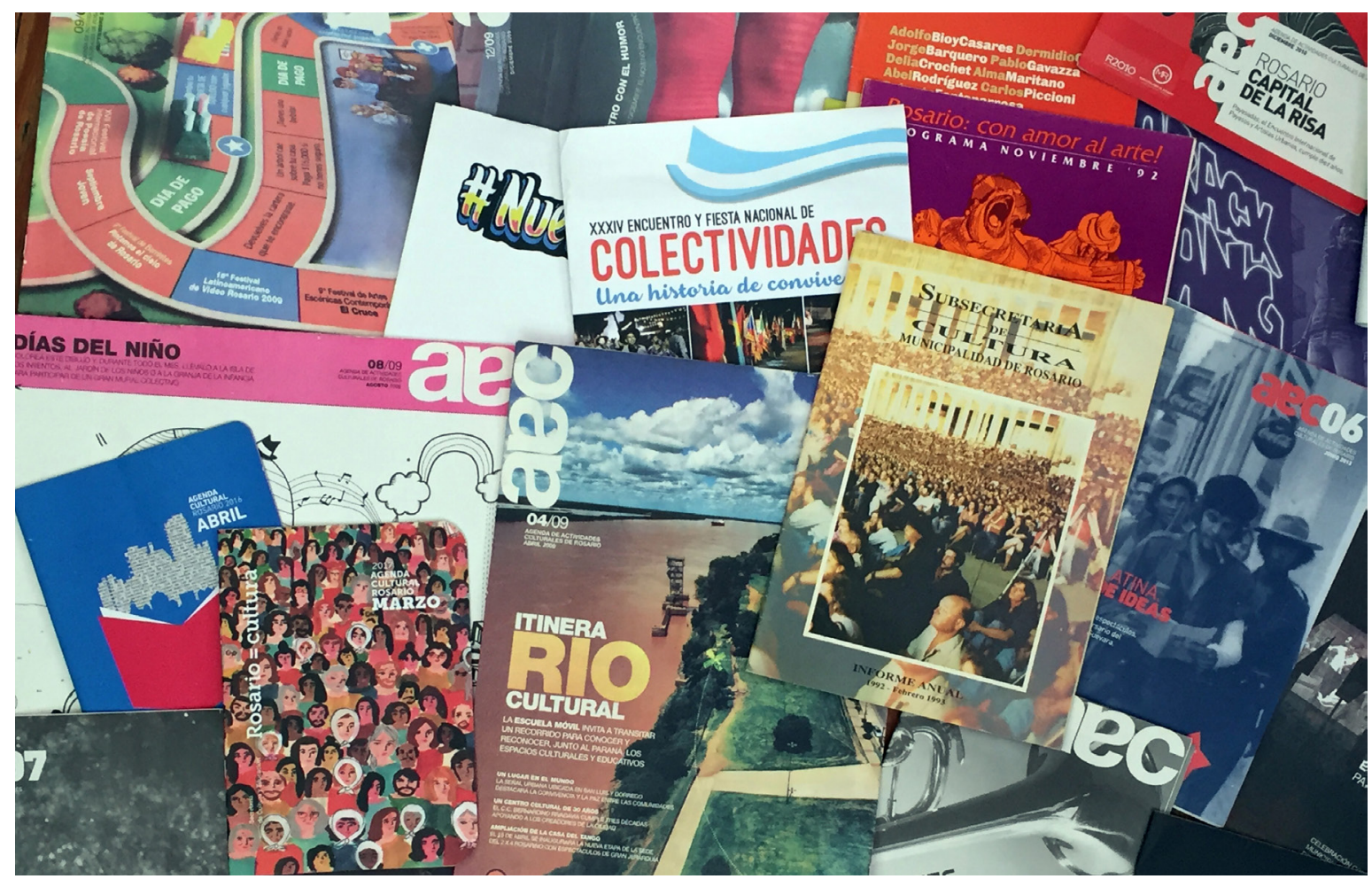

LUCIANO OMINETTI - Agendas e informes de actividades culturales de la ciudad de Rosario.

decirse que la gestión cultural pasó a manos de reconocidos referentes de la ciudad, no sólo por el prestigio de sus obras intelectuales sino también por el compromiso con los valores antiautoritarios" (2000: 245).

El nuevo programa cultural se situó en antagonismo con el periodo precedente, con la realización de actividades al aire libre, espectáculos masivos en plazas de distintos barrios, la contratación de músicos y actores locales, y la edición de una revista que por primera vez registró las actividades culturales de la ciudad. ${ }^{7}$ Se realizaron eventos feriales regionales, y se impulsaron talleres barriales y centros culturales en zonas alejadas del foco político-administrativo y comercial rosarino.

Hasta ese momento, desde el accionar estatal, prevalecían ciertas concepciones elitistas en términos de la cultura, a la que se entendía como lo culto, el arte, un producto espiritual y superior. Sin embargo, se introdujeron otras perspectivas por medio de modificaciones de las actividades y en el marco de ese espíritu de época que intentaba distanciarse del periodo anterior. En ese contexto de transición y apertura, hubo una fuerte orientación hacia la participación popular, el pluralismo y la democracia trasladada a las producciones culturales, ya fueran musicales, literarias, teatrales o plásticas, entre otras. Esas concepciones estuvieron teñidas de la necesidad de afianzar las instituciones, en tanto misión de restablecer una noción del Estado que "sea de todos" y recuperar la pertenencia de lo público, en

La revista Vasto Mundo, editada por la Subsecretaría de Cultura municipal, era de distribución gratuita e incluía historietas, notas y entrevistas a distintas personas vinculadas con el ámbito cultural de la ciudad. 
confrontación con la percepción heredada del periodo dictatorial del Estado como enemigo (entrevista con Julio Rayón, Rosario, 28 de noviembre de 2013). Al mismo tiempo, lo cultural estaba asociado a lo artístico y podía funcionar como relleno o complemento, matices que expresaban sus sentidos utilitaristas: "servía siempre y cuando apoyara a una idea política" (entrevista con Rafael Ielpi, Rosario, 2 de marzo de 2011). Mientras tanto, en esos años también se planteó la posibilidad de que la acción cultural se "abriera a los barrios" y se reconociera "el tejido urbano de Rosario como mucho más amplio" que el histórico centro político-administrativo de la ciudad (entrevista con Alberto Callaci, Rosario, 17 de enero de 2013).

\section{La década de 1990 y la nueva jerarquía burocrática}

Desde 1989, con Héctor Cavallero (1989-1991; 1991-1995) al frente, el gobierno municipal pasó por primera vez a manos del Partido Socialista Popular. En ese periodo, la Subsecretaría de Cultura estuvo a cargo, primero, de Ada Donato (1989-1991), profesora de letras y escritora; luego, de Enrique Llopis (1992-1993), un reconocido músico, y finalmente, de Héctor Pichi de Benedictis (1993-1995), otro importante músico de la ciudad. Bajo la expresión "la cultura de Rosario está conducida por hombres de la cultura", el intendente presentaba a los referentes de las áreas culturales del Estado municipal. ${ }^{8}$

En la década de 1990 se acentuaron las modificaciones en las concepciones de las acciones, que ya se hacían perceptibles con la apertura democrática. Por eso, en esos años, se reformuló el organigrama con nuevas dependencias: Área de Educación, Artes Visuales, Bibliotecas, Música y Área de Organismos Externos; también se crearon la Dirección de Promoción Cultural y las Casas de Cultura Barrial, además de que se propagaron los talleres barriales de

la década anterior. ${ }^{9}$ En 1992 se creó la Editorial Municipal, desde la cual se iniciaron proyectos de publicación de libros, revistas y discografía. ${ }^{10}$ En otro orden, se establecieron las primeras normativas para la regularización de espacios feriales preexistentes.

En 1993, la Subsecretaría de Cultura, dependiente de la Secretaría de Gobierno y Cultura, pasó al rango de Secretaría de Cultura, Educación y Turismo, por primera vez con presupuesto propio. Fue un momento clave de las políticas culturales municipales, cuando, pese a las dificultades socioeconómicas que padecía la ciudad, este ámbito estatal asumió una nueva jerarquía.

El cambio en el organigrama institucional puede ser entendido como un avance y un reconocimiento del área cultural, parte sustantiva de las políticas públicas municipales. Las concepciones de lo cultural presentes en los periodos anteriores se intensificaron en términos de "herramienta" para afianzar una gestión de gobierno determinada, tal como lo expresan algunos funcionarios: "[la política] siempre la usó a la cultura, para sus fines" (entrevista con Héctor Pichi de Benedictis, Rosario, 22 de diciembre de 2014). En algunos momentos de la historia del país y de la ciudad, la concepción de cultura como "circo", "para darle alegría a la gente", pretendió funcionar como

8

9

tabo
taba con tres museos, tres teatros, dos bibliotecas, cuatro
escuelas - museología, danzas, arte escénico y música-, seis organismos estables, una editorial, el servicio educativo, una Dirección de Promoción Cultural de la cual dependían nueve talleres de participación barrial, dos centros culturales, tres casas de cultura y tres dependencias del Complejo Astronómico Educativo (Municipalidad de Rosario-Subsecretaría de Cultura, 1993).

10 En 1989 se sanciona la Ordenanza 4.700/89 de creación de la editorial municipal, que se reglamenta en julio de 1992 y se amplía en la Ordenanza 5.427/92. Su primer director fue el escritor rosarino Héctor Sebastianelli. Véase Honorable Concejo Deliberante de Rosario (1989; 1992). 
una pantalla de distracción. Asimismo, mientras esas concepciones subsistían, se producía una apertura hacia la idea de "ganar el espacio público" (entrevista con Héctor Pichi de Benedictis, Rosario, 22 de diciembre de 2014). Mientras se redefinían los sentidos de lo cultural con elementos de deselitización, democratización y participación, en algunas áreas seguían presentes las nociones tradicionales de cultura como "arte", "vidriera" o "espectáculo".

Volviendo al hecho de que el Estado dotara a las políticas culturales de anclaje formal para su formulación como política pública mediante el establecimiento de una dependencia específica, otra cara de este proceso supuso cierta precarización, pues mientras adquiría su nuevo papel debió administrar los mismos recursos: "el presupuesto es más magro", "cambia el nivel, pero en cada paso se pierden cosas" (entrevista con Julio Rayón, Rosario, 28 de noviembre de 2013). En ese "perder" y "ganar" cosas, las áreas estatales de cultura se expandieron y adquirieron mayor presencia en las políticas públicas del municipio, en un contexto económico y social sumamente crítico. En el ámbito nacional, la década de 1990 estuvo marcada por las medidas de corte neoliberal, con ajustes macroeconómicos que introdujeron reformas institucionales como la descentralización de responsabilidades y funciones del gobierno nacional hacia las provincias, principalmente en las áreas de educación, salud y política social (Manzanal, 2006).

A partir de 1995, la intendencia de la ciudad pasó al frente de otro referente del Partido Socialista, Hermes Binner (1995-1999; 19992003). En este periodo, el secretario de Cultura fue, primero, Héctor Tealdi (1995-1998), un destacado actor y director de la ciudad, y más adelante, Marcelo Romeu (1998-2003), un integrante del partido en el gobierno. Durante esta administración se creó la Agenda Cultural Rosarina, que concentró las actividades culturales mensuales desarrolladas en diferentes puntos de la ciudad, y el
Programa de Descentralización y Modernización de la Municipalidad de Rosario, que llevó adelante la descentralización político-administrativa de la ciudad en seis distritos. Este proceso se consolidó a finales de la década de 1990 y a principios de la siguiente, con base en el tamaño de la ciudad, la cantidad de habitantes y la "existencia de desequilibrios que se hace necesario compensar" (PER, 1998).

Esta tendencia estructural tuvo un camino específico desde el gobierno local, que desembocó en diversos debates sobre cómo llevar a cabo la descentralización. ¿Cómo acercar el Estado a la gente? ¿Qué sucedería con el "lugar del génesis [el centro políticoadministrativo tradicional de la ciudad], iba a morir"? (entrevista con Julio Rayón, Rosario, 28 de noviembre de 2013). En la formulación y puesta en práctica de este nuevo modelo territorial se condensaron concepciones divergentes respecto a la idea de ciudad y la posibilidad de generar nuevos centros que se salieran del foco político y comercial tradicional.

De la mano de la descentralización políticoadministrativa que comenzó a finales de la década de 1990, se crearon las áreas de cultura en las cabeceras distritales, que resignificaron la territorialidad de las acciones, al sumar o superponer actividades en los barrios abarcados por las nuevas delimitaciones políticas. Aunque las delimitaciones propuestas para la organización de los distritos tuvieron en cuenta aspectos históricos de la conformación de la ciudad -Centro, Norte, Noroeste, Oeste, Sudoeste y Sur-, no siempre coincidían con las fronteras geográficas y culturales de sus habitantes. Asimismo, esta diversificación de las dependencias y acciones produjo superposición de las propuestas, lo que expresaba la falta de una política articulada. Esta tensión puso en evidencia cierto "distribucionismo cultural" hacia barrios distantes del centro político-administrativo tradicional (García, 1987), pero esto no apaciguó las diferencias en las formas de producción y apropiación de la cultura producto de la desigualdad socioeconómica. 


\section{Las primeras décadas del siglo XXI y la nueva geografía urbana}

Desde 2000 en adelante prosiguió la ampliación y complejización de las dependencias, programas y proyectos culturales. Si bien la creación de las nuevas áreas descentralizadas supuso una redefinición del trabajo en materia cultural en diversas zonas, a partir de 2001 y 2002 se produjo un repliegue hacia el centro tradicional de la ciudad, con actividades espectaculares que no alcanzaban al público de los barrios y distritos distantes. Para estos últimos se impulsaron programas y acciones relacionados con emprendimientos productivos, subsidios y planes de desempleo, ligados a la economía social o solidaria, que se impusieron por encima de la actividad cultural interrumpida por la crisis. ${ }^{11}$

Durante las siguientes gestiones, con el gobierno de Miguel Lifschitz (2003-2007; 2007-2011), continuaron propagándose nuevas áreas, dependencias y programas, hasta quedar configurada finalmente la Secretaría de Cultura y Educación. En esos años, la dependencia estuvo a cargo de Marina Naranjo (2003-2006), profesora y educadora; después le siguieron María de los Ángeles Chiqui González (2006-2007), directora y docente de teatro; Fernando Farina (2007-2009), crítico y artista vinculado a las artes plásticas, y Horacio Ríos (2010-2015), un referente dentro de la producción audiovisual.

En la actualidad, la ciudad tiene 46 dependencias, que incluyen bibliotecas públicas, museos, centros culturales, escuelas de arte, salas y teatros, y espacios para la infancia, ${ }^{12}$ con una planta total de 1026 trabajadores pertenecientes a las áreas culturales municipales (Municipalidad de Rosario, s.f.).

Al mismo tiempo, mientras se extendía el proceso de descentralización, el gobierno local se concentró en otros espacios, asociados a una nueva geografía cultural: la costa de la ciudad, que supuso ocupar áreas hasta entonces obsoletas, afectadas por el antiguo puerto, como los viejos galpones desocupados que en ese momento estaban en el centro de las disputas políticas, económicas y simbólicas. El paisaje costero se prefiguró en la representación imaginaria de los observadores que proyectaban la ciudad como el "cordón cultural", el "circuito de mercados y ferias muy rosarinas", en un proceso de reutilización y emblematización de esos espacios de Rosario en los que la cultura pasó a ocupar un lugar significativo como recurso de apropiación y reconstrucción de identidades. Asimismo, esas modificaciones en las significaciones y usos del espacio se relacionaron con las transformaciones atravesadas por la propia ciudad, vinculadas a lo que algunos autores denominan el "reencantamiento urbano", que apuntaba al entretenimiento, el ocio y la estimulación del consumo (Amendola, 2000, citado en País, 2016).

\section{La expansión del horizonte de la cultura}

Entrelazadas en el movimiento de complejización detectado en los organigramas institucionales y normativos, algunas de las nociones que en ellos se utilizan permiten identificar elementos de una concepción ampliada de lo cultural. Esto se hizo patente en la deselitización expresada en la propagación de nuevas producciones, prácticas y actores que se incorporaron a la escena de lo cultural desde la pluralidad y la diversidad. Las concepciones elitistas y esencialistas de la cultura siguieron vigentes en distintos ámbitos pero su hegemonía dentro de las acciones culturales locales fue disputada.

11 Entre 2003 y 2004 se crearon nuevas dependencias en el área de promoción social municipal, tales como la Subsecretaría de Economía Solidaria y demás programas de emprendimientos productivos.

12 Dirección General de Entidades y Organismos; Dirección General de Programas Educativos y Tríptico de la Infancia; Dirección General de Programación Cultural y Dirección General de Descentralización Cultural (Honorable Concejo Deliberante de Rosario, 2008; Municipalidad de Rosario, s.f.). 
El devenir de las gestiones en materia de cultura estuvo marcado por la injerencia de los productores culturales de la ciudad, protagonistas de renovaciones y posturas críticas. Salvo excepciones, escritores, poetas, músicos, actores y artistas plásticos ocuparon lugares de decisión y formulación de las políticas culturales estatales. Esto se se relaciona con los cruces entre el Estado y los movimientos de producción cultural voluntaria, que conducen a preguntarse: ¿cuáles fueron las concepciones y prácticas que marcaron los pulsos del campo cultural?, ¿cómo se ubicó lo cultural, lo político y el papel del Estado? Estas dimensiones pueden ser rastreadas por medio del análisis de lo recabado, en términos de las trayectorias de gran parte de los funcionarios a la hora de ocupar cargos de decisión altos en las áreas culturales del municipio, puesto que fueron convocados para asumir cargos en el Estado mientras contaban con gran reconocimiento o eran referentes en los campos artísticoculturales a los cuales pertenecían - literatura, música, dramaturgia, teatro, artes plásticas—. ${ }^{13}$ De ello surge una serie de lineamientos sobre cómo se entiende lo público, que se entrelaza con los recorridos personales, familiares, políticos, artísticos e ideológicos, así como con los contextos políticos de gobierno que convocaron a estos actores a asumir puestos altos en materia de cultura.

En las entrevistas, el hecho de ocupar cargos de decisión se relacionó con una concepción del Estado y de la tarea que debían desempañar aquellos que participaron en distintos campos de la producción cultural de la ciudad. ${ }^{14}$ Para varios de los funcionarios que trabajaron en la década de 1990, había que ocupar los espacios estatales: "meter la mano en el Estado", restituirlo, porque "es de todos" y "es un trabajo que hay que hacer", "un deber que hay que hacer" (entrevista con Héctor Pichi de Benedictis, Rosario, 22 de diciembre de 2014). Otros funcionarios atribuían las razones de su decisión a la confluencia de la "formación teórica", el contacto con "cuadros políticos" y las organizaciones barriales, que permitieron dar un "salto importante" para asumir el desafío de ingresar en el área estatal (entrevista con Enrique Llopis, Rosario, 3 de septiembre de 2015). Tal como enuncia una funcionaria: "haber empezado [su] vida creativa en medio de la dictadura", mezclada con la participación política, movimientista de la década de 1970, "era el ejercicio de la propia acción política y de la acción cultural dentro de la acción política" (entrevista con María de los Ángeles Chiqui González, Rosario, 14 de enero de 2014).

En la década de 1990, trabajar en el Estado significaba la posibilidad de tener acceso a un empleo en plena crisis laboral, y al mismo tiempo, ocupar ese espacio permitía "transformar el mundo desde allí" (entrevista con Luis Alfonso, Rosario, 31 de julio de 2017). En palabras de este trabajador del área cultural:

Yo nunca me sentí como un empleado municipal, porque justamente [...] yo crecí pensando que uno debía transformar el mundo, que era una condición del ser humano hacer que el mundo sea mejor. Entonces, desde los 13, 14 años, siempre tuve algún grado de militancia, siempre fue a nivel de lo cultural [...] en el 76, 77, 78 laburábamos [...]

Un aspecto específico del campo cultural rosarino se relaciona con la dificultad de trazar fronteras entre los trabajadores de áreas estatales, los propios funcionarios y los agrupamientos artísticos. En ocasiones se dan pertenencias simultáneas a sectores estatales y llamados independientes o autogestivos, en distintos tipos de producción cultural y artística.

14 Las personas entrevistadas suelen recuperar lo político, lo social, lo administrativo y lo artístico como las esferas de su tarea. Por su complejidad, el trabajo en las áreas culturales supone el desarrollo de diversas cualidades, entre las cuales se mencionan la capacidad de observación de las realidades locales, el establecimiento de criterios estéticos y artísticos, el involucramiento político, la administración de recursos o la falta de ellos. Estas habilidades se enuncian como condiciones para el desempeño del trabajo, pero no siempre confluyen y pueden prevalecer unas sobre otras. 
como se podía, ¿no?, antidictatorial. O sea que, de alguna manera, cuando empecé a trabajar acá, sentí que recuperaba un espacio, por el cual nunca antes me habían pagado, que ahora me pagaban un sueldo y yo podía seguir haciendo lo que siempre hice.

Las referencias de los actores a su experiencia evocan la propia práctica articulada a sus militancias políticas previas.

El conocimiento del lugar de los productores culturales, ya sea en relación con la música, la literatura, el teatro, las artes plásticas u otras, en algunos casos planteó una visión desde dentro de los agrupamientos que se resignificó una vez que ocuparon puestos en el Estado y se confrontó con las concepciones y demandas de los distintos sectores, expresando las dificultades de tramitar institucionalmente las disputas del propio campo.

La deselitización conceptual implicó un viraje hacia propuestas de transformación social y emancipación presentes en los discursos y prácticas de algunos de los funcionarios de la época, que representan continuidades con las acciones de las décadas anteriores y que a partir de la década de 1990 pesaron como los motores de la función pública y el trabajo en el Estado. Pero ese énfasis en la democratización cultural no quedó al margen de las tendencias hegemónicas que comenzaron a ubicar lo cultural como recurso y herramienta, no sólo como vía de transformación e inclusión, sino también para su inserción en el mercado. Las dinámicas instrumentales orientaron lo cultural según los intereses en juego de los distintos contextos y los protagonistas de las contiendas entre el Estado, los sectores independientes y los propios públicos. Para la experiencia analizada, las tendencias generales se expresaron en la centralidad de lo cultural como medio de transformación, crecimiento y desarrollo urbano, y se alternaron con las orientaciones hacia la cultura del entretenimiento, los consumos y la mercantilización de las producciones culturales y artísticas.

\section{Consideraciones finales}

$\mathrm{Al}$ poner en consideración las transformaciones que se produjeron en la institucionalidad organizacional de las estructuras del Estado municipal para desarrollar su política en el ámbito de la cultura (Garretón, 2001), arribamos a momentos clave de las políticas culturales en Rosario. De manera simultánea a las innovaciones en la institucionalidad normativa, el área cambió su denominación en consonancia con lo que le sucedió a las políticas y a la ciudad: en 1983, de una Dirección de Cultura dependiente del gobierno se pasó a una Subsecretaría de Cultura, Turismo y Educación; en 1993, la subsecretaría se constituyó en Secretaría de Cultura, Turismo y Educación; en 2000, se formó la Secretaría de Cultura y Educación. Las políticas públicas culturales se asociaron a las esferas educativas y turísticas. Mientras se expandían las franjas de esparcimiento y ocio para el consumo y la promoción urbana, en otras zonas de la ciudad se exacerbaban las dificultades de acceso a los servicios sociales, educativos, de transporte y vivienda.

La periodización que propusimos partió de finales de la década de 1980 y el análisis nos condujo a identificar a la década de 1990 como significativa y paradójica. En ese contexto, el país atravesó un periodo neoliberal, con medidas político-económicas como la flexibilización laboral, la privatización de servicios públicos y la concentración de actividades bancarias y financieras, mientras se producían desempleos masivos y se profundizaban la pobreza y las desigualdades sociales, lo que desembocó en la crisis de 2001. En el transcurso de esos años se produjeron cambios sustantivos en la institucionalidad del sector cultural en Rosario. ¿Sería acaso la composición del campo cultural de esa ciudad la respuesta para ese proceso? Las condiciones particulares del campo cultural de la ciudad, con una diversidad de grupos artísticos; la apuesta de los gobiernos municipales de convocar a los propios productores como 
protagonistas de las áreas estatales de cultura, y el empuje de los movimientos artístico-culturales pueden ser parte de las respuestas a esa interrogante. Pese a las dificultades del contexto general y local, trabajar en el Estado se inscribió también en las concepciones de transformación social heredadas de prácticas políticas de las décadas anteriores, que aun interpeladas por la coyuntura eran una vía de salida ante la crisis. Al mismo tiempo, las tendencias hegemónicas internacionales que instalaron lo cultural como recurso para el desarrollo se impusieron también en esa década, de modo que pudieron haber incidido en algunas de las formulaciones de las acciones del periodo.

Luego de recorrer los itinerarios de las políticas públicas culturales de Rosario en el contexto argentino, sostenemos que la dimensión del trabajo es sustancial para comprender las articulaciones entre cultura y política. Mediante el análisis de las concepciones y prácticas de los actores significativos de esas políticas, pudimos rastrear cómo confluyeron sus trayectorias formativas en el campo cultural y político de la ciudad, como aspecto específico del entramado institucional seleccionado.

A finales de 2017, la orientación de las políticas públicas nacionales deja ver los elementos de las tendencias neoliberales que se expanden en la región: reducción del gasto público, restricción de los presupuestos en diversas áreas - principalmente en cultura, educación e investigación científica一, reformas fiscales, previsionales y laborales que restringen los derechos de los trabajadores, entre otras..$^{15}$ Mediante una concentración oligopólica en la prensa, la radio y la televisión, el gobierno controla la opinión pública. Esto plantea un nuevo punto de inflexión en todas las esferas de las políticas públicas marcadas por el avance neoliberal. Analizar las dimensiones del impacto de esa nueva configuración políticoinstitucional será tarea de próximas indagaciones sobre los alcances de esas medidas para la ciudad de Rosario, en el contexto de la sociedad argentina y latinoamericana. D

15

\section{Bibliografía}

Achilli, Elena, 1998, "Vivir en la pobreza urbana. El derecho a una interculturalidad no excluyente”, en Revista Lote, núm. 18. Disponible en línea: <http://www.fernandopeirone.com.ar/Lote/nro018/achilli.html>.

Águila, Gabriela, 2000, “El terrorismo de Estado sobre Rosario (1976-1983)”, en Alberto Pla (coord.), Rosario en la historia (de 1930 a nuestros días), tomo 2, Universidad Nacional de Rosario Editora, Rosario, pp. 121-221.

Aguilar Criado, Encarnación, 2005, "Patrimonio y globalización: el recurso de la cultura en las políticas de desarrollo europeas", en Cuadernos de Antropología Social, núm. 21, pp. 51-69.

Álvarez, Juan, 1998 [1943], Historia de Rosario (1689-1939), Universidad Nacional de Rosario Editora/Editorial Municipal de Rosario, Rosario. Arantes, Augusto (org.), 1984, Produzindo o passado. Estratégias de construção do patrimônio cultural, Editora Brasiliense, São Paulo.

Bayardo, Rubens, 2000, "Antropología, identidad y políticas culturales", en Programa Antropología de la Cultura, Instituto de Ciencias Antropológicas-Facultad de Filosofía y Letras-Universidad de Buenos Aires, Buenos Aires.

- 2010, "Políticas culturales y derechos: entre la retórica y la realidad", en Revista de Investigaciones Políticas y Sociológicas, vol. 9 , núm. 2, pp. 55-64.

Bonfil Batalla, Guillermo, 1987, “Los pueblos indios, sus culturas y las políticas culturales”, en Néstor García Canclini (ed.), Políticas culturales en América Latina, Grijalbo, México, pp. 89-125. 
Brunner, José, 1987, “Políticas culturales y democracia: hacia una teoría de las oportunidades”, en Néstor García Canclini (ed.), Politicas culturales en América Latina, Grijalbo, México, pp. 175-203.

Cardini, Laura Ana, 2011, Los usos de la cultura. Un análisis de las políticas culturales en Rosario (1990-2010), proyecto de la carrera de investigador del Consejo Nacional de Investigaciones Científicas y Técnicas, Investigaciones Sociohistóricas Regionales, Rosario.

__ 2013, "Políticas culturales y patrimonio en la ciudad de Rosario, Argentina”, en Boletín de Antropología, vol. 28, núm. 46, pp. 124-142.

Cardini, Laura Ana y David Madrigal González (comps.), 2018, Cultura, antropología y transformación social desde las politicas culturales de México, Brasil y Argentina, El Colegio de San Luis, San Luis Potosí.

Chaui, Marilena, 2008, "Cultura y democracia", en Cuadernos del Pensamiento Crítico Latinoamericano, núm. 5. Disponible en línea: <http:// bibliotecavirtual.clacso.org.ar/ar/libros/secret/cuadernos/es/cha.pdf>.

Ceballos, Rita (ed.), 1989, Antropología y políticas culturales. Patrimonio e identidad, Talleres Gráficos de la Dirección Nacional del Registro Oficial, Buenos Aires.

Crespo, Carolina, Hernán Morel y Margarita Ondelj (comps.), 2015, La política cultural en debate: diversidad, performance y patrimonio cultural, Ediciones Ciccus, Buenos Aires.

Honorable Concejo Deliberante de Rosario, 1989, Ordenanza 4.700/89, Creación de la Editorial Municipal, Rosario. , 1992, Ordenanza 5.427/92. Formalización de la creación de la Editorial Municipal, Rosario.

2008, Decreto 371, Secretaría de Cultura y Educación. Nueva Estructura Orgánica, Boletín Oficial 118, Rosario.

Documental Programa Cultural en Barrios de Rosario 1992-1993, Edición: diciembre de 1996.

Durham, Eunice, 1984, "Texto II", en Augusto Arantes (org.), Produzindo o passado. Estratégias de construção do patrimônio cultural, Editora Brasiliense, São Paulo, pp. 23-58.

Fernández, Sandra y Marisa Armida, 2000, “Una ciudad en transición y crisis (1930-1943)", en Alberto Pla (coord.), Rosario en la historia (de 1930 a nuestros días), tomo 2, Universidad Nacional de Rosario Editora, Rosario, pp. 23-151.

García Canclini, Néstor (ed.), 1987, Políticas culturales en América Latina, Grijalbo, México.

Garretón, Manuel (coord.), 2001, “I. Cultura y espacio cultural en el mundo globalizado”, en El espacio cultural latinoamericano. Bases para una politica cultural de integración, Fondo de Cultura Económica/Convenio Andrés Bello, México, pp. 19-36.

Getino, Octavio, 1995, "Políticas públicas y legislación”, en Las políticas culturales en la Argentina, Ediciones Colihue, Buenos Aires, pp. $339-352$.

Guevara, Gustavo, 2000, "Rosario en los ochenta y los noventa”, en Alberto Pla (coord.), Rosario en la historia (de 1930 a nuestros días), tomo 2, Universidad Nacional de Rosario Editora, Rosario, pp. 223-331.

Hardoy, Jorge, 1985, “La vivienda obrera en una ciudad en expansión. Rosario entre 1858 y 1910”, en Richard Morse y Jorge Hardoy (comps.), Cultura urbana latinoamericana, Consejo Latinoamericano de Ciencias Sociales, Buenos Aires, pp. 63-93.

Instituto Nacional de Estadísticas y Censos (INDEC), 2011, Censo Nacional de Población, Hogares y Viviendas 2010, Aglomerado Rosario, Rosario. Landi, Oscar, 1987, “Campo cultural y democratización en Argentina”, en Néstor García Canclini (ed.), Políticas culturales en América Latina, Grijalbo, México, pp. 145-173.

Lifschitz, Laura, 2008, “La Guía Quincenal o de la inserción de la cultura letrada en el primer gobierno peronista”, en Actas del Primer Congreso de Estudios sobre el Peronismo: la Primera Década, Mar del Plata. Disponible en línea: <http://redesperonismo.com.ar/archivos/CD1/SC/ lifschitz.pdf>.

Logiódice, María Julia, 2012, "La Agrupación Discepolín. Articulaciones entre política y teatro en Rosario durante los ochenta”, en Telón de Fondo. Revista de Teoría y Crítica Teatral, núm. 16, pp. 102-116.

Manzanal, Mabel, 2006, "Descentralización y municipios en Argentina. Contrastes y contradicciones", en Crítica en Desarrol/o, Revista Latinoamericana de Ciencias Sociales, núm. 1, pp. 37-60.

Miceli, Sergio, 1987, “Estado, mercado y necesidades populares: las políticas culturales en Brasil”, en Néstor García Canclini (ed.), Políticas culturales en América Latina, Grijalbo, México, pp. 127-143.

Municipalidad de Rosario, s.f., Página institucional de la Secretaría de Cultura y Educación. Disponible en línea: <http://www.rosariocultura. gob.ar/la-secretaria>. Consultado el 9 de marzo de 2011.

Municipalidad de Rosario-Subsecretaría de Cultura, 1993, Informe Anual 1992-1993, Rosario.

Municipalidad de Rosario-Secretaría General, 1998, La descentralización en Rosario, 1998. Programa de Descentralización y Modernización, Rosario.

Naranjo, Rubén (ed.), 1973, Paraná, el pariente del mar, Departamento de publicaciones de la Biblioteca Popular Constancio C. Vigil, Rosario. 
Niño Amieva, Alejandra, 2007, “Instituciones culturales, discurso e identidad”, en Adversus, año IV, núms. 8-9. Disponible en línea: <http:// www.adversus.org/indice/nro8-9/dossier/dossier_ninoamieva.htm\#nota17>.

Ochoa, Ana María, 2002, "Políticas culturales, academia y sociedad”, en Daniel Mato (coord.), Estudios y otras prácticas intelectuales latinoamericanas en cultura y poder, Consejo Latinoamericano de Ciencias Sociales/Comisión de Estudios de Posgrado/Facultad de Ciencias Económicas y Sociales-Universidad Central de Venezuela, Caracas, pp. 213-224.

País Andrade, Marcela, 2016, Identidades culturales en y desde las fronteras. Un enfoque de género a la(s) política(s) y a la(s) práctica(s) culturales juveniles, Antropofagia, Buenos Aires.

Plan Estratégico Rosario (PER), 1998, Programa de autonomía local, modernización y descentralización municipal, Rosario.

Secretaría de Cultura de la Nación, 1984, Plan Nacional de Cultura 1984-1989, Buenos Aires.

, 1990, Plan Federal de Cultura, Buenos Aires.

Raggio, Liliana, 2015, “Los derechos culturales. Estado, medios de comunicación y organizaciones de la sociedad civil”, en Mónica Rotman (comp.),

Dinámicas de poder. Procesos patrimoniales, politicas y gestión de la cultura, Filo/Universidad de Buenos Aires, Buenos Aires, pp. $211-241$.

Rotman, Mónica, 1996, "Política cultural, gestión municipal y prácticas artesanales", en Publicar, año 5, núm. 6, pp. 47-67.

Vich, Víctor, 2014, Desculturizar la cultura. La gestión cultural como forma de acción política, Siglo XXI Editores, Buenos Aires.

Yúdice, George, 2002, El recurso de la cultura. Usos de la cultura en la era global, Gedisa, Barcelona.

Yúdice, George y Toby Miller, 2004, "Introducción: historia y teoría de la política cultural”, en Política cultural, Gedisa, Barcelona, pp. 11-53.

Zukin, Susan, 1995, The Cultures of Cities, Blackwell, Oxford.

\section{Entrevistas}

Alberto Callaci, Rosario, 17 de enero de 2013.

Enrique Llopis, Rosario, 3 de septiembre de 2015.

Héctor Pichi de Benedictis, Ente Turístico de Rosario, Municipalidad de Rosario, 22 de diciembre de 2014.

Julio Rayón, Rosario, 28 de noviembre de 2013.

Luis Alfonso, Dirección General de Programación, Secretaría de Cultura y Educación, Municipalidad de Rosario, 31 de julio de 2017.

María de los Ángeles Chiqui González, Ministerio de Innovación e Cultura, Gobierno de la Provincia de Santa Fe, Rosario, 14 de enero de 2014. Rafael Ielpi, Centro Cultural Roberto Fontanarrosa, Municipalidad de Rosario, 2 de marzo de 2011.

\section{Filmografía}

Programa Cultural en Barrios de Rosario 1992-1993, documental, diciembre de 1996.

\section{Anexo}

Denominación de las dependencias culturales nacionales

a) Comisión Nacional de Cultura, 1933

b) Secretaría de Cultura de la Nación, 1946.

c) Subsecretaría de Cultura 1948 (dependiente del Ministerio de Educación; la Comisión Nacional de Cultura pasa a ser un órgano consultivo).

d) Dirección Nacional de Cultura 1950 (reglamentada en 1954).

e) Secretaría de Cultura, 1984.

f) Ministerio de Cultura de la Nación, 2014.

Denominación de las dependencias culturales municipales

a) Dirección de Cultura 1937 (dependiente de la Secretaría de Gobierno de la Municipalidad de Rosario).

b) Subsecretaría de Cultura 1983 (dependiente de la Secretaría de Gobierno y Cultura de la Municipalidad de Rosario).

c) Secretaría de Cultura, Educación y Turismo de la Municipalidad de Rosario, 1993.

d) Secretaría de Cultura y Educación de la Municipalidad de Rosario, 2000. 